\title{
Fulminant heart failure due to giant cell myocarditis affecting the left ventricle
}

\author{
Tomasz Senderek ${ }^{1}$, Barbara Małecka ${ }^{1,2}$, Andrzej Ząbek ${ }^{1}$, Lucyna Rudnicka-Sosin ${ }^{3}$, Karol Wierzbicki ${ }^{2,4}$, \\ Piotr Przybyłowski ${ }^{2,4}$, Jacek Bednarek ${ }^{1}$, Jacek Lelakowski ${ }^{1,2}$ \\ ${ }^{1}$ Department of Electrocardiology, John Paul II Hospital, Krakow, Poland \\ ${ }^{2}$ Institute of Cardiology, Jagiellonian University, Medical College, Krakow, Poland \\ ${ }^{3}$ Department of Patomorphology, John Paul II Hospital, Krakow, Poland \\ ${ }^{4}$ Department of Cardiosurgery, John Paul II Hospital, Krakow, Poland
}

Postep Kardiol Inter 2015; 11, 4 (42): 351-353

DOI: $10.5114 /$ pwki.2015.55613

\begin{abstract}
A bstract
A 56-year-old woman, previously healthy, was hospitalized after an episode of ventricular tachycardia in the course of infection. In view of the fulminant course of heart failure the patient was connected to an extracorporeal membrane oxygenation (ECMO) system. After 3 weeks of treatment with ECMO the patient received a heart transplant. A histopathological examination of the tissues of the explanted heart revealed giant cell myocarditis. The patient was treated with immunosuppression based on induction therapy followed by a standard regimen with steroids. Currently, the patient remains in good general condition with an left ventricular ejection fraction of $60 \%$.
\end{abstract}

Key words: fulminant heart failure, giant cell myocarditis.

\section{Case report}

A 56-year-old woman, previously healthy, was hospitalized after an episode of ventricular tachycardia (VT) in the course of infection manifested by weakness and cough. The transthoracic echocardiography (TTE) showed reduced left ventricular ejection fraction (LVEF) to $40 \%$. Laboratory tests indicated changed parameters of inflammation (leukocytosis $12.27 \times 10^{3} / \mu \mathrm{l}$ ), levels of brain natriuretic peptide (NT-proBNP) elevated to $6241 \mathrm{pg} / \mathrm{ml}$, and a lower level of potassium $(3.2 \mathrm{mmol} / \mathrm{l})$.

Seventy-two hours after hospitalization, diagnostics of acute coronary syndrome due to persistent elevated levels of troponin to 0.54 (ng/ml) was performed. Coronarography showed no changes in the coronary arteries but further reduction of LVEF to $15 \%$.

The diagnostic algorithm and therapeutic options in heart failure were taken into consideration [1].

Tachyarrhythmic cardiomyopathy in the course of recurrent atrial fibrillation with a fast (up to $180 / \mathrm{min}$ ) ventricular rate and episodes of non-sustained VT was suspected (Figures 1, 2). Because of the ineffectiveness of pharmacotherapy, it was decided to perform ablation of the AV node preceded by implantation of a pacing system. During the VVI pacemaker implantation procedure there were problems in obtaining proper pacing and sensing parameters. Ultimately the septal right ventricular outflow tract was chosen for the endocardial lead location.

A day after surgery the patient twice underwent cardiac arrest in the course of ventricular fibrillation. Gradual deterioration of electrical parameters of cardiac pacing and further reduction of LVEF to $5-10 \%$ were noted. NT-proBNP increased to $11113 \mathrm{pg} / \mathrm{ml}$ with normal levels of procalcitonin and the negative results of tests for Lyme disease and enterovirus. In view of the fulminant course of heart failure the patient was connected to an extracorporeal membrane oxygenation (ECMO) system. The biopsy of the right ventricular muscle showed no inflammatory lesions [2]. After 3 weeks of treatment with ECMO the patient received a heart transplant. A histopathological examination of the tissues of the explanted heart revealed giant cell myocarditis with areas of cardiomyocyte necrosis and formation of fibrous scar limited to the walls of the left ventricle and the interventricular septum (Figure 3). The patient was treated with immu-

\section{Corresponding author:}

Andrzej Ząbek MD, MSc, Department of Electrocardiology, John Paul II Hospital, 80 Prądnicka St, 31-202 Krakow, Poland, phone: +48 694194 757, e-mail: andrzej___z@poczta.onet.pl

Received: 29.10.2014, accepted: 23.02.2015. 


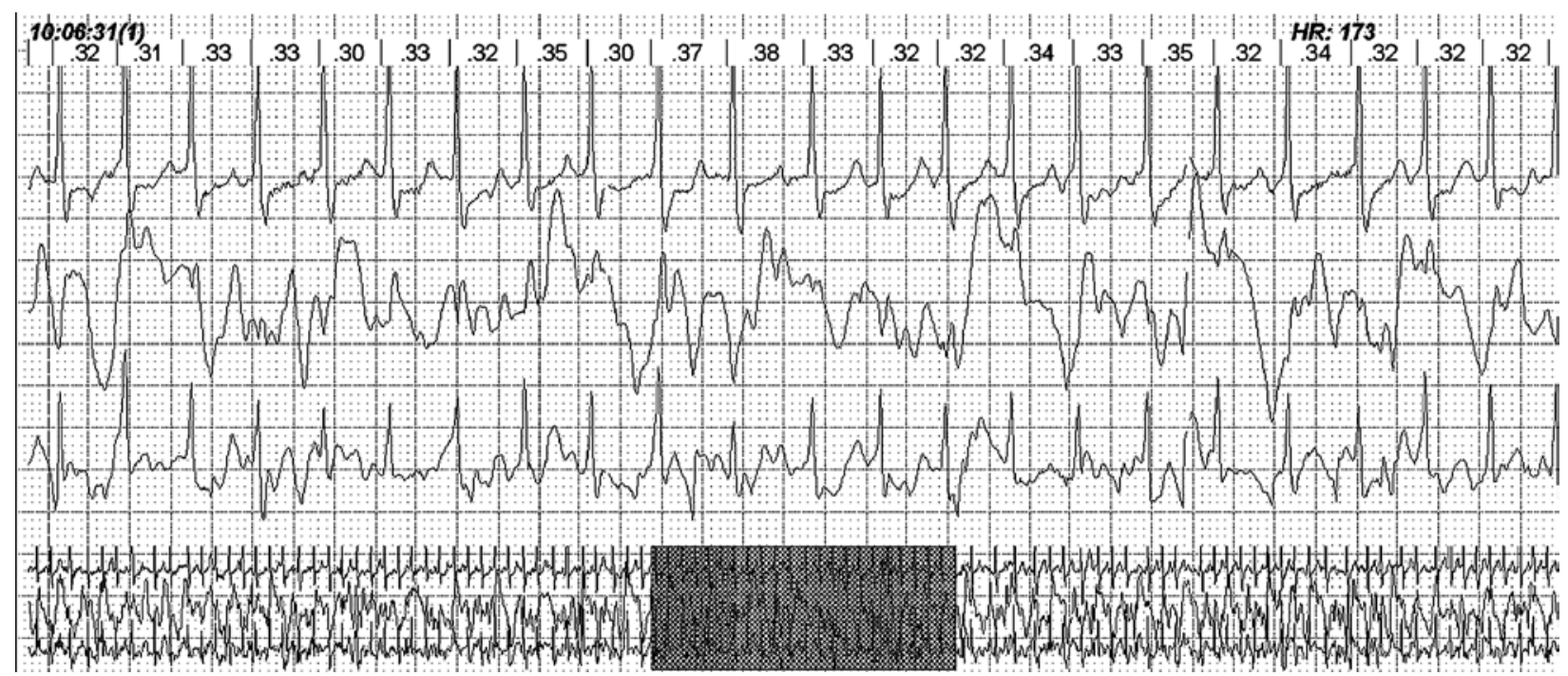

Figure 1. Holter ECG: atrial fibrillation with fast ventricular rate

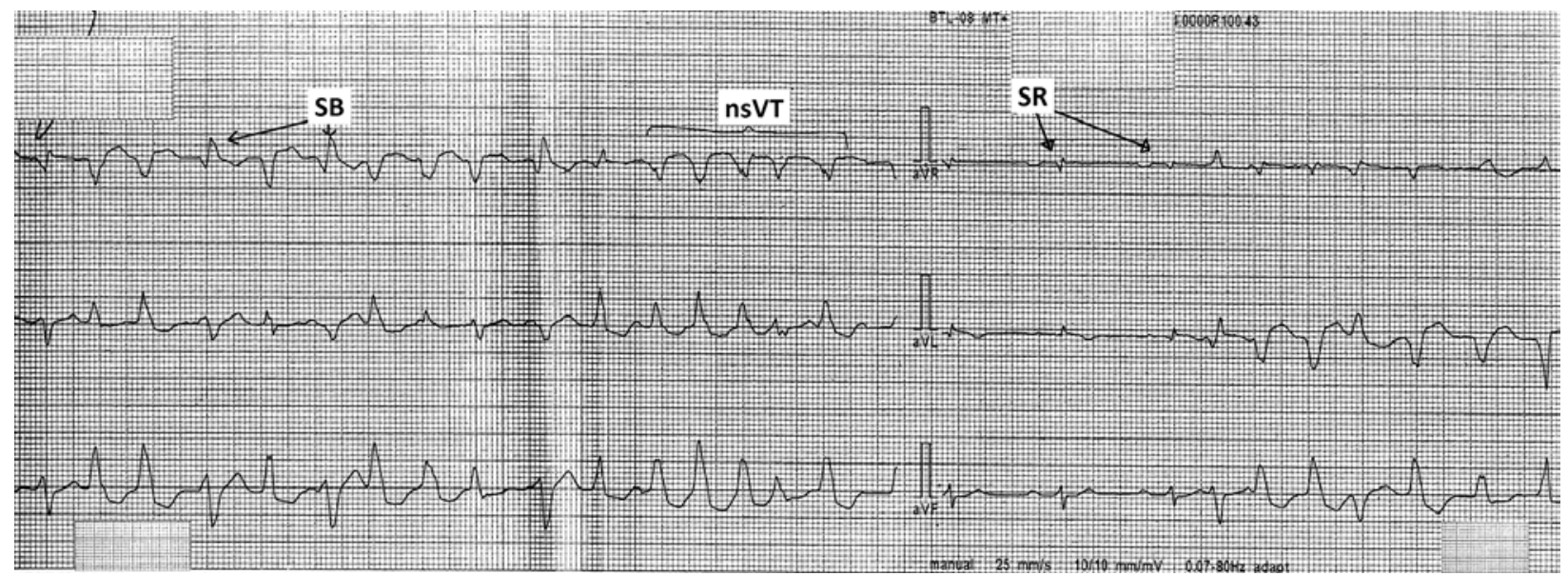

Figure 2. ECG: arrows show: sinus beats (SR), supraventricular beats (SB), episodes of non-sustained VT (nsVT)

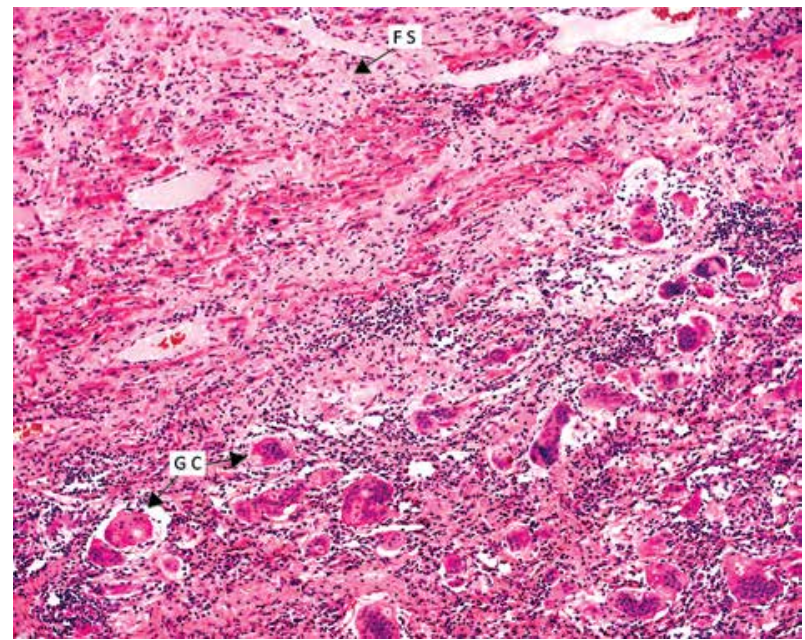

Figure 3. Histopathological examination: specimens from the wall of the left ventricle and interventricular septum - image of giant cell (GC) myocarditis with areas of fresh and degradable organization with the formation of fibrous scar (FS) tissue nosuppression based on induction therapy followed by a standard regimen with steroids. Currently, the patient remains in good general condition with an LVEF of $60 \%$. All protocolar biopsies showed no signs of rejection or other histological abnormalities including recurrence of myocarditis.

\section{Discussion}

Giant cell myocarditis is a rare disease with a poor prognosis [3, 4]. It leads to progressive congestive heart failure, with ventricular arrhythmias not treatable by pharmacology or ablation. Some patients have symptoms resembling acute myocardial infarction or complete heart block. The diagnosis is made on the basis of the histological image.

In that case the heart biopsy should be taken earlier, although it is not always crucial. Due to a lack of strong signs of inflammation or any other reason explaining the fulminant course, the wrong diagnosis of tachyarrhythmic cardiomyopathy was made. It was followed by first- 
step treatment with implantation of a pacemaker. During that surgery electrical parameters in the septum were not fully proper because of giant cells in the left ventricle and septum. In the case of lack of a left ventricle assist device (LVAD) only ECMO before heart transplantation can help.

Ninety percent of patients die within a few weeks or have a heart transplantation, which is the best chance to save the patient's life. The disease process develops in around $30 \%$ of grafted hearts and it can be stopped by immunosuppressants including steroids.

\section{Conflict of interest}

The authors declare no conflict of interest.

\section{References}

1. Gajos G. Diagnostic algorithm and therapeutic options in chronic heart failure: updated review of clinical practice guidelines. Pol Arch Med Wewn 2008; 118: 489-500.

2. Luk A, Metawee M, Ahm E, et al. Do clinical diagnoses correlate with pathological diagnoses in cardiac transplant patients? The importance of endomyocardial biopsy. Can J Cardiol 2009; 25: e48-54.

3. Cooper LT Jr, Berry GJ, Shabetai R. Idiopathic giant-cell myocarditis - natural history and treatment. N Engl J Med 1997; 336: 1860-6.

4. Suzuki J, Ogawa M, Watanabe R, et al. Autoimmune giant cell myocarditis: clinical characteristics, experimental models and future treatments. Expert Opin Ther Targets 2011; 15: 1163-72. 